Article

\title{
Exact Solutions for Time-Dependent Non-Hermitian Oscillators: Classical and Quantum Pictures
}

\author{
Kevin Zelaya ${ }^{1, * \mathbb{D}}$ and Oscar Rosas-Ortiz ${ }^{2}$ D \\ 1 Nuclear Physics Institute, The Czech Academy of Science, 25068 Řež, Czech Republic \\ 2 Physics Department, Cinvestav, AP 14-740, México City 07000, Mexico; orosas@fis.cinvestav.mx \\ * Correspondence: zelayame@crm.umontreal.ca
}

Citation: Zelaya, K.; Rosas-Ortiz, O. Exact Solutions for Time-Dependent Non-Hermitian Oscillators: Classical and Quantum Pictures. Quantum Rep. 2021, 3, 458-472. https://doi.org/ 10.3390 /quantum3030030

Academic Editor: J. Gonzalo Muga

Received: 22 July 2021

Accepted: 18 August 2021

Published: 21 August 2021

Publisher's Note: MDPI stays neutral with regard to jurisdictional claims in published maps and institutional affiliations.

Copyright: (c) 2021 by the authors. Licensee MDPI, Basel, Switzerland. This article is an open access article distributed under the terms and conditions of the Creative Commons Attribution (CC BY) license (https:/ / creativecommons.org/licenses/by/ $4.0 /)$.

\begin{abstract}
We associate the stationary harmonic oscillator with time-dependent systems exhibiting non-Hermiticity by means of point transformations. The new systems are exactly solvable, with all-real spectra, and transit to the Hermitian configuration for the appropriate values of the involved parameters. We provide a concrete generalization of the Swanson oscillator that includes the Caldirola-Kanai model as a particular case. Explicit solutions are given in both the classical and quantum pictures.
\end{abstract}

Keywords: point transformations; non-Hermiticity; time-dependent phenomena; exact solutions; Caldirola-Kanai oscillator; Swanson oscillator; Ermakov equation

\section{Introduction}

During the last decades, there has been an increasing interest in non-Hermitian structures and their implications in quantum theory. Nevertheless, the subject was overlooked for long time because the reality of the spectrum is not granted a priori for non-Hermitian models. A surprising breakthrough was offered by the demonstration that parity-time (PT) symmetry implies the real spectrum [1], stimulating the systematic search of PT-symmetric systems in quantum mechanics [2] (see also papers in the special issues [3,4]). Further improvements have shown that PT-symmetry is not a necessary condition for the reality of the spectrum [5,6] - a fact confirmed in diverse models of non-Hermiticity [7-11], where the imaginary part of a wide class of complex-valued potentials leads to balanced gain and loss probability without the necessity of PT-symmetry. The latter property opens new possibilities in optical design, where the gain-loss manipulation includes but is not limited to PT-symmetry [12]. Important theoretical achievements within the PT-symmetric formulation [13] predicted the experimental observation of the phenomenon in optics [14].

Another interesting problem deals with non-stationary systems in quantum mechanics, which find exciting applications in plasma physics [15] as well as in the design of electromagnetic traps for charged particles [16-21]. As time-dependent systems obey dynamical equations that cannot be reduced to eigenvalue problems in general, finding solutions could mean a formidable consumption of computing resources. Two simplifications are notable: systems obeying adiabatic evolutions [22] and the Lewis-Riesenfeld (parametric) oscillator [23]. In the former case, the time-evolution of the system is slow enough for the Hamiltonian to tolerate time-dependent eigenvalues for instantaneous eigenvalue equations [24]. In turn, the parametric oscillator is found to have a constant of motion that defines the appropriate eigenvalue equation [23]. The latter result motivated the systematic research of quantum invariants [25-28], with applications in the construction of time-dependent wave packets [29-37], Darboux transformations [38-41], and two-dimensional photonic systems [42], among others.

The Swanson oscillator [43] is a very peculiar system that combines both profiles since it is non-Hermitian and time-dependent. Formulated to study transitions of probability amplitudes that are generated by non-unitary time evolutions, the model developed by 
Swanson has been revisited and studied in different branches of physics and mathematical physics [44-50]. Quite remarkably, the Swanson Hamiltonian can be connected with the Hamiltonian of the harmonic oscillator by the appropriate rotation in configuration space [51], which clarifies the solvability of the model.

In this work, we use point transformations [52] to associate the stationary harmonic oscillator with a time-dependent system that exhibits non-Hermiticity and an all-real spectrum. We have already applied the method to study parametric oscillators in the Hermitian regime [27], and we have also shown that the construction of coherent states is feasible for such systems. Here, we show that the appropriate point transformation yields a wide family of time-dependent systems that may be chosen to be Hermitian or non-Hermitian, in accordance with the involved parameters. Associating these systems with the stationary harmonic oscillator, we obtain exactly solvable models that recover a diversity of oscillators in both Hermitian and non-Hermitian configurations.

The organization of the paper is as follows. In Section 2, we introduce the main concepts and provide the space of solutions for the non-Hermitian, time-dependent systems in terms of the well known solutions of the harmonic oscillator. Concrete expressions are given for the Hamiltonian of such systems. In Section 3, we show the applicability of our model by solving the dynamical law of a generalization of the Caldirola-Kanai oscillator in both classical and quantum pictures. We have added Appendix A in which the point transformation theory is summarized.

\section{Generalized Oscillators}

Let us assume that the operator

$$
\frac{1}{\hbar w_{0}} H_{\mathrm{sw}}=\alpha_{2}(t) \hat{a}^{\dagger 2}+\beta_{2}(t) \hat{a}^{2}+\theta(t)\left\{\hat{a}, \hat{a}^{\dagger}\right\}+\alpha_{1}(t) \hat{a}^{\dagger}+\beta_{1}(t) \hat{a},
$$

rules the dynamical law of a time-dependent quantum system. The constant $w_{0}>0$ is written in units of frequency. Hereafter, $\hat{a}$ and $\hat{a}^{\dagger}$ represent the boson ladder operators fulfilling $\left[\hat{a}, \hat{a}^{\dagger}\right]=\mathbb{I}$, with $\mathbb{I}$ the identity operator in the Hilbert space spanned by the Fock basis $\{|n\rangle, n=0,1,2, \ldots\}$. The symbols $[\cdot, \cdot]$ and $\{\cdot, \cdot\}$ stand for the commutator and anticommutator of the involved operators, respectively.

The structure of $H_{\mathrm{sw}}$ resembles the expression of the Hamiltonian for the Swanson oscillator $[43,47,53,54]$. Nevertheless, subtle but relevant differences must be noted since the coefficients $\alpha_{j}(t), \beta_{j}(t), j=1,2$, and $\theta(t)$ are time-dependent functions introduced to design the profile of $H_{\mathrm{sw}}$ in Equation (1). Indeed, we distinguish four important configurations:

(I) Harmonic oscillator: Making $\alpha_{j}=\beta_{j}=0$ and $\theta=\frac{1}{2}$, the operator (1) is reduced to the well known Hamiltonian of the harmonic oscillator:

$$
\frac{1}{\hbar w_{0}} H_{\mathrm{osc}}=\frac{1}{2}\left\{\hat{a}, \hat{a}^{\dagger}\right\}=\hat{a}^{\dagger} \hat{a}+\frac{1}{2} .
$$

(II) Hermitian configuration: For $\theta \in \mathbb{R}$, and $\alpha_{j}, \beta_{j} \in \mathbb{C}$ such that $\beta_{j}=\alpha_{j}^{*}$, with $z^{*}$ the complex conjugate of $z \in \mathbb{C}$, the operator $H_{\mathrm{sw}}$ is Hermitian. The harmonic oscillator Hamiltonian (2) is properly included in this class.

(III) Global non-Hermitian configuration: In general, for arbitrary complex-valued functions $\alpha_{j}, \beta_{j}$, and $\theta$, the operator (1) is non-Hermitian. The two cases mentioned above are therefore relevant subclasses of this configuration.

(IV) Non-Hermitian configuration: A subset of the global non-Hermitian class is characterized by real coefficients $\alpha_{j}, \beta_{j}$, and $\theta$. This includes the harmonic oscillator as well as a subset of the Hermitian classes.

We are interested in solving the Schrödinger equation defined by $H_{\mathrm{sw}}$. In the most general situation, no orthogonality is granted a priori for the solutions as both timedependence and non-Hermiticity bring the problem out of the Sturm-Liouville formalism. In the same context, the boson operators $\hat{a}$ and $\hat{a}^{\dagger}$ are not necessarily the ladder operators for the corresponding set of solutions. 
Without loss of generality, we consider the Hermitian and non-Hermitian classes as they are described above. The analysis of the global non-Hermitian case is provided elsewhere, as it requires a more elaborate treatment.

The operator $H_{\mathrm{sw}}$ may be expressed in terms of the quadratures of position and momentum. If one uses the well known relationships

$$
\hat{a}=\sqrt{\frac{m_{0} w_{0}}{2 \hbar}}\left(\hat{x}+\frac{i}{m_{0} w_{0}} \hat{p}_{x}\right), \quad \hat{a}^{\dagger}=\sqrt{\frac{m_{0} w_{0}}{2 \hbar}}\left(\hat{x}-\frac{i}{m_{0} w_{0}} \hat{p}_{x}\right),
$$

then (1) acquires the quadratic form

$$
H_{\mathrm{SW}}(t)=\frac{\hat{p}_{x}^{2}}{2 m(t)}+\frac{m(t) w^{2}(t)}{2} \hat{x}^{2}+i \Omega(t)\left\{\hat{x}, \hat{p}_{x}\right\}+i v(t) \hat{p}_{x}+F(t) \hat{x},
$$

where the linear terms are characterized by the time-dependent functions

$$
\Omega(t):=-w_{0}\left[\alpha_{2}(t)-\beta_{2}(t)\right], \quad v(t):=-\sqrt{\frac{\hbar w_{0}}{2 m_{0}}}\left[\alpha_{1}(t)-\beta_{1}(t)\right],
$$

and

$$
F(t):=\sqrt{\frac{m_{0} \hbar w_{0}^{2}}{2}}\left[\alpha_{1}(t)+\beta_{1}(t)\right] .
$$

The mass and frequency terms of (4) are also time-dependent

$$
m(t)=\frac{m_{0}}{2 \theta(t)-\left[\alpha_{2}(t)+\beta_{2}(t)\right]}, \quad w^{2}(t)=w_{0}^{2}\left(4 \theta^{2}(t)-\left[\alpha_{2}(t)+\beta_{2}(t)\right]^{2}\right) .
$$

Note that the above formulae are defined by the expressions $\alpha_{j} \pm \beta_{j}$, which facilitates their identification according to the classification provided above. Next, we provide explicit expressions.

Before proceeding, one may revert the previous relationships and write down the parameters of the bosonic representation (1) in terms of those of the quadrature representation (4). We thus obtain

$$
\begin{aligned}
& \alpha_{2}(t)=\frac{m^{2}(t) w^{2}(t)-m_{0}^{2} w_{0}^{2}-2 m_{0} w_{0} m(t) \Omega(t)}{4 m_{0} w_{0}^{2} m(t)}, \\
& \beta_{2}(t)=\frac{m^{2}(t) w^{2}(t)-m_{0}^{2} w_{0}^{2}+2 m_{0} w_{0} m(t) \Omega(t)}{4 m_{0} w_{0}^{2} m(t)},
\end{aligned}
$$

together with

$$
\begin{aligned}
& \alpha_{1}(t)=\frac{-m_{0} \sqrt{w_{0}} v(t)+F(t)}{\sqrt{2 \hbar m_{0} w_{0}^{2}}}, \quad \beta_{1}(t)=\frac{m_{0} \sqrt{w_{0}} v(t)+F(t)}{\sqrt{2 \hbar m_{0} w_{0}^{2}}}, \\
& \theta(t)=\frac{m_{0}^{2} w_{0}^{2}+m^{2}(t) w^{2}(t)}{4 m_{0} w_{0}^{2} m(t)} .
\end{aligned}
$$

- Hermitian configuration: For $\beta_{j}=\alpha_{j}^{*}$, systems (5) and (6) give

$$
i \Omega=\Omega_{I}:=2 w_{0} \operatorname{Im}\left(\alpha_{2}\right), \quad i v=v_{I}:=\sqrt{\frac{2 \hbar w_{0}}{m_{0}}} \operatorname{Im}\left(\alpha_{1}\right), \quad F_{R}=\sqrt{2 m_{0} \hbar w_{0}^{2}} \operatorname{Re}\left(\alpha_{1}\right),
$$

together with

$$
m_{R}=\frac{m_{0}}{2 \theta-2 \operatorname{Re}\left(\alpha_{2}\right)}, \quad w_{R}^{2}=4 w_{0}^{2}\left(\theta^{2}+\left[\operatorname{Re}\left(\alpha_{2}\right)\right]^{2}\right) .
$$


Then, (4) is written in the self-adjoint form:

$$
\widetilde{H}_{\mathrm{sw}}(t)=\frac{\hat{p}_{x}^{2}}{2 m_{R}(t)}+\frac{m_{R}(t) w_{R}^{2}(t)}{2} \hat{x}^{2}+\Omega_{I}(t)\left\{\hat{x}, \hat{p}_{x}\right\}+v_{I}(t) \hat{p}_{x}+F_{R}(t) \hat{x} .
$$

Clearly, there is a one-to-one correspondence between the sets $\mathcal{S}_{\text {bos }}=\left\{\alpha_{1}, \alpha_{2}, \beta_{1}, \beta_{2}, \theta\right\}$ and $\mathcal{S}_{\text {quad }}=\{m, w, \Omega, v, F\}$. Thus, by fixing the parameters in $\mathcal{S}_{\text {bos }}$, one can determine the parameters in $\mathcal{S}_{\text {quad }}$, and vice versa.

- Non-Hermitian configuration: For real coefficients $\alpha_{j}, \beta_{j}$, and $\theta$, Equations (4)-(7) yield $H_{\mathrm{sw}}$ such that $H_{\mathrm{sw}}^{+} \neq H_{\mathrm{sw}}$. The non-Hermiticity is due to the real-valued functions $\Omega$ and $v$, which may be cancelled by making $\alpha_{j}=\beta_{j}$. Noticeably, the latter case is consistent with the Hermitian configuration mentioned in the previous item after making $\Omega_{I}=v_{I}=0$. For $\Omega_{I} \neq 0$ and $v_{I} \neq 0$, the appropriate transformation shows that, providing $\Omega=v=0$, the self-adjoint operator $\widetilde{H}_{\mathrm{sw}}$ coincides with $H_{\mathrm{sw}}$.

Considering the above remarks, the model is developed within the non-Hermitian configuration defined by the real coefficients $\alpha_{j}, \beta_{j}$, and $\theta$. The Hermitian configuration will be recovered after making $\alpha_{j}=\beta_{j}$. On the other hand, without loss of generality, hereafter, we make $F=0$. From (6), the latter implies $\beta_{1}=-\alpha_{1}$, so that $v=\sqrt{\frac{2 \hbar w_{0}}{m_{0}}} \beta_{1}$.

\subsection{Space of Solutions}

In position representation, the solutions of the Schrödinger equation defined by the Hamiltonian of the harmonic oscillator (2) are well known to be

$$
\Psi_{n}(y, \tau)=e^{-i E_{n} \tau} \Phi_{n}(y), \quad E_{n}=\hbar w_{0}\left(n+\frac{1}{2}\right), \quad n=0,1, \ldots,
$$

where $\tau$ stands for the time-variable, and the normalized functions

$$
\Phi_{n}(y)=\sqrt{\frac{1}{2^{n} n !} \sqrt{\frac{m_{0} w_{0}}{\pi \hbar}}} e^{-\frac{m_{0} w_{0}}{2 \hbar} y^{2}} H_{n}\left(\sqrt{\frac{m_{0} w_{0}}{\hbar}} y\right),
$$

satisfy the eigenvalue equation $H_{\mathrm{Osc}} \Phi_{n}(y)=E_{n} \Phi_{n}(y)$, with $H_{n}(z)$ the Hermite polynomials [55].

To construct the solutions of the Schrödinger equation defined by $H_{\mathrm{sw}}$, we use the approach introduced in [27], which is based on the formalism of point transformations. Detailed information is provided in Appendix A. Using $t$ and $x$ for the time-variable and position-coordinate of the system governed by $H_{\mathrm{sw}}$, the transformation

$$
\tau(t)=\int^{t} \frac{d t^{\prime}}{\sigma^{2}\left(t^{\prime}\right)}, \quad y(x, t)=\frac{\mu(t) x+\gamma(t)}{\sigma(t)}, \quad m(t)=m_{0} \mu^{2}(t),
$$

permits us to express the solutions we are looking for in terms of the formulae (13) and (14). The time-dependent functions $\gamma(t)$ and $\sigma(t)$ are to be determined.

Within the point transformation approach, the rules (15) lead to a time-dependent potential in the $(x, t)$-configuration. Explicitly,

$$
V(x, t)=\frac{m_{0} \mu^{2}}{2}\left(\frac{\dot{\mathcal{W}}_{\mu}}{\mu \sigma}+\frac{2 i}{\mu^{2}} \frac{d}{d t}\left(\mu^{2} \Omega\right)-4 \Omega^{2}+\frac{w_{0}^{2}}{\sigma^{4}}\right) x^{2}+m_{0} \mu\left(\frac{\dot{\mathcal{W}}_{\gamma}}{\sigma}-2 i \mu \Omega v+\frac{i}{\mu} \frac{d}{d t}\left(\mu^{2} v\right)+\frac{w_{0}^{2} \gamma}{\sigma^{4}}\right) x+V_{0}(t),
$$

with $\mathcal{W}_{\mu}$ and $\mathcal{W}_{\gamma}$ being two functions of time defined in Equation (A11), and $V_{0}(t)$ is given in Equation (A12) of Appendix A.

In turn, the wave-functions $\psi_{n}(x, t)$ are given by

$$
\psi_{n}(x, t)=e^{-i E_{n} \tau(t) / \hbar} \phi_{n}(x, t),
$$


where the functions $\phi_{n}(x, t)$ are constructed through the eigenfunctions of the stationary $(y, \tau)$-system:

$$
\phi_{n}(x, t)=A_{0}^{-1}(x, t) A_{1}^{-1}(x, t) \Phi_{n}(y(x, t)),
$$

with

$$
\begin{aligned}
& A_{0}(x, t)=\exp \left[i \frac{m_{0}}{\hbar} \frac{\mu}{\sigma}\left(\frac{\mathcal{W}_{\mu}}{2} x^{2}+\mathcal{W}_{\gamma} x+\xi_{1}\right)\right] \\
& A_{1}(x, t):=\exp \left[-\frac{m_{0}}{\hbar} \mu^{2}\left(\Omega x^{2}+v x+\xi_{2}\right)\right]
\end{aligned}
$$

and

$$
\frac{\mu}{\sigma} \xi_{1}=\frac{\gamma W_{\gamma}}{2 \sigma}+\int^{t} d t^{\prime}\left(\frac{\mu^{2}\left(t^{\prime}\right) v^{2}\left(t^{\prime}\right)}{2}-\mu\left(t^{\prime}\right) v\left(t^{\prime}\right) \Omega\left(t^{\prime}\right) \gamma\left(t^{\prime}\right)\right) .
$$

Thus, providing the solutions (13) and (14) of the stationary $(y, \tau)$-system, we automatically obtain the solutions (17)-(20) of a time-dependent system in the $(x, t)$-configuration.

Note that we have conveniently introduced the form of $\psi$ given in (17) so that the timedependent factor $e^{-i E_{n} \tau(t) / \hbar}$ can be immediately identified with the phase introduced by Lewis-Riesenfeld [23] for the parametric oscillator. In this form, the point transformation provides a straightforward mechanism to determine this factor, which is nothing but the transformation of the unitary time-evolution phase of the stationary oscillator. Alternative approaches for non-Hermitian Hamiltonians have been previously studied in [53].

We would also like to emphasize that the expression of $V(x, t)$ introduced in Equation (16) represents a wide resource of complex-valued, time-dependent potentials linked to the stationary harmonic oscillator. The applicability of the above results is therefore very wide. This embraces time-dependent Hermitian oscillators for real-valued functions $V(x, t)$ as well as non-Hermitian oscillators (stationary and non-stationary) for complex-valued functions $V(x, t)$. The main point is the manipulability of the concrete form of $V(x, t)$ by tuning its time-dependent coefficients.

In the present work, we concentrate on the relationship between $V(x, t)$ and the potential part of $H_{\mathrm{Sw}}$, written in coordinate representation (4). Other oscillators will be studied elsewhere.

\subsection{Time-Dependent Model with Non-Hermiticity}

Comparing the non-kinetic part of $H_{\mathrm{sw}}$ with $V(x, t)$ leads to Equations (A13)-(A15) of Appendix A. The time-dependent functions $\gamma$ and $\sigma$ that define the transformation (15) are solutions of (A13) and (A14), respectively. These equations include pure-imaginary terms that may be canceled through the constraints

$$
\mu^{2} \Omega=\Omega_{0}, \quad \mu^{2} v=v_{0},
$$

where $\Omega_{0}$ and $v_{0}$ are real constants to be fixed. The functions $\gamma$ and $\sigma$ are therefore defined by the following system of equations:

$$
\ddot{\sigma}+\left(w^{2}+4 \frac{\Omega_{0}^{2}}{\mu^{4}}-\frac{\ddot{\mu}}{\mu}\right) \sigma=\frac{w_{0}^{2}}{\sigma^{3}}, \quad \ddot{\gamma}+\left(w^{2}+4 \frac{\Omega_{0}^{2}}{\mu^{4}}-\frac{\ddot{\mu}}{\mu}\right) \gamma=2 \frac{v_{0} \Omega_{0}}{\mu^{3}},
$$

together with

$$
\frac{d}{d t}\left(\frac{\mu}{\sigma} \xi_{1}+i \mu^{2} \xi_{2}-\frac{\gamma}{2 \sigma} \mathcal{W}_{\gamma}-i \frac{\hbar}{2 m_{0}} \ln \frac{\mu}{\sigma}\right)-\frac{v_{0}^{2}}{2 \mu^{2}}+v_{0} \Omega_{0} \frac{\gamma}{\mu^{3}}-\frac{\hbar}{m_{0}} \frac{\Omega_{0}}{\mu^{2}}=0 .
$$

The real-valued functions $\xi_{1}(t)$ and $\xi_{2}(t)$ resulted from integration with respect to $x$. Note that the equation for $\sigma$ in (23) has the structure of the nonlinear differential equation named after Ermakov [56]. Detailed information about the method of solution and applications can be found in, e.g., $[7,9,57]$. 
On the other hand, combining (22) with (8) and (9) gives rise to the set of parameters $\mathcal{S}_{\text {bos }}$ of the bosonic representation (1). Therefore, the operator introduced in Equation (4) acquires a simpler form

$$
H_{\mathrm{sw}}(t)=\frac{\hat{p}_{x}^{2}}{2 m_{0} \mu^{2}(t)}+\frac{m_{0} \mu^{2}(t) w^{2}(t)}{2} \hat{x}^{2}+\frac{i}{\mu^{2}(t)}\left[\Omega_{0}\left\{\hat{x}, \hat{p}_{x}\right\}+v_{0} \hat{p}_{x}\right],
$$

where $\mu$ plays the role of a time-dependent mass.

The non-Hermiticity of $H_{\mathrm{sw}}$ in Equation (25) is parameterized by the real constants $\Omega_{0}$ and $v_{0}$; the Hermitian version of this operator arises by turning off both of these parameters. Paying attention to the transformation of the position-variable (15), we realize that $y(x, t)$ is real-valued if the $x$-coordinate is real, as expected. The construction of non-Hermitian operators $H_{\mathrm{sw}}$ permitting complex-valued mappings for $y(x, t)$ may be derived from a more general scheme, which is out of the scope of the present work.

Hereafter, we take $\Omega_{0} \geq 0$ and $v_{0} \geq 0$. The complete characterization of the operator (25) is therefore provided by the analytical form of the time-dependent functions $\alpha_{2}$ and $\beta_{2}$. Remarkably, up to the non-Hermitian term, the expression of $H_{\mathrm{sw}}$ in (25) has the structure of the generalized Caldirola-Kanai oscillator discussed in [58] within the Arnold transformation approach. Here, the operator (25) is linked with a time-dependent oscillator in the nonHermitian regime for which both mass and frequency depend on time. As the Hermitian limit $\Omega_{0}=v_{0}=0$ has already been treated in $[58,59]$, it is discarded throughout the rest of the manuscript.

\section{Applications}

To illustrate the applicability of our approach, we consider a model generated by the time-dependent functions

$$
\mu^{2}=e^{-\Gamma t}, \quad w^{2}=w_{0}^{2}
$$

The Formulas (8) and (9) give

$$
\begin{aligned}
& \alpha_{2}(t)=-\frac{1}{2} \sinh (\Gamma t)-\frac{\Omega_{0}}{2 w_{0}} e^{\Gamma t}, \quad \beta_{2}(t)=-\frac{1}{2} \sinh (\Gamma t)+\frac{\Omega_{0}}{2 w_{0}} e^{\Gamma t}, \\
& \theta=\frac{1}{2} \cosh (\Gamma t), \quad \beta_{1}=\sqrt{\frac{m_{0}}{2 \hbar w_{0}}} v_{0} e^{\Gamma t} .
\end{aligned}
$$

Therefore, the operator (25) acquires the form

$$
H_{\mathrm{sW}}(t)=\frac{e^{\Gamma t}}{2 m_{0}} \hat{p}_{x}^{2}+\frac{m_{0} w_{0}^{2}}{2} e^{-\Gamma t} \hat{x}^{2}+i e^{\Gamma t}\left(\Omega_{0}\left\{\hat{x}, \hat{p}_{x}\right\}+v_{0} \hat{p}_{x}\right) .
$$

The self-adjoint part of this operator coincides with the Hamiltonian studied independently by Caldirola [60] and Kanai [61]. That is, the Hamiltonian $H_{\mathrm{Sw}}(t)$ introduced in Equation (28) may be considered a non-Hermitian extension of the Caldirola-Kanai oscillator.

At the classical level, the Caldirola-Kanai Hamiltonian leads to the Newton equation of motion, including a friction term that is proportional to velocity. This property motivated the unfinished debate about the nature and possible interpretation of friction forces in quantum mechanics [62]. Considered a very interesting problem in the formal structure of quantum mechanics, the Caldirola-Kanai oscillator deserves particular attention. For instance, it has been studied in terms of the quantum Arnold transformation [26,58], where exact solutions have been provided for the related Schrödinger equation.

Considering the interest that non-Hermitian structures such as the Hamiltonian (28) arouse in the literature on the matter, we solve the corresponding dynamical law for both the classical and quantum pictures. 


\subsection{Classical Picture}

To determine the classical equations of motion associated to the classical counterpart of operator (28), consider the classical Hamiltonian

$$
H_{\text {class }}(Q, P ; t)=\frac{e^{\Gamma t} P^{2}}{2 m_{0}}+\frac{e^{-\Gamma t} m_{0} w_{0}^{2}}{2} Q^{2}+i e^{\Gamma t}\left(2 \Omega_{0} Q P+v_{0} P\right) .
$$

Using canonical quantization, together with the symmetrization rule $Q P \rightarrow \frac{1}{2}\left\{\hat{x}, \hat{p}_{x}\right\}$, operator (28) is recovered, as expected.

The Hamilton equations of motion, $\dot{Q}=\frac{\partial H_{\text {class }}}{\partial P}$ and $-\dot{P}=\frac{\partial H_{\text {class }}}{\partial Q}$, yield

$$
P=m_{0} e^{-\Gamma t} \dot{Q}-2 i m_{0}\left(\Omega_{0} Q+v_{0}\right),
$$

which is complex-valued in the configuration space $(Q, \dot{Q})$. From (30), we have $\dot{Q}=$ $e^{\Gamma t}\left(m_{0}^{-1} P+2 i\left(\Omega_{0} Q+v_{0}\right)\right)$, so that $\ddot{Q}=\left\{\dot{Q}, H_{\text {class }}\right\}_{\mathrm{PB}}+\frac{\partial \dot{Q}}{\partial t}$, where $\{\cdot, \cdot\}_{\mathrm{PB}}$ stands for the Poisson bracket. After some calculations, one arrives at equation

$$
\ddot{Q}-\Gamma \dot{Q}+\left(w_{0}^{2}+4 \Omega_{0}^{2} e^{2 \Gamma t}\right) Q=-2 \Omega_{0} v_{0} e^{2 \Gamma t},
$$

which defines the behavior of a damped parametric oscillator with a time-dependent frequency $\left(w_{0}^{2}+4 \Omega_{0}^{2} e^{2 \Gamma t}\right)$ and that is subjected to the force $-2 \Omega_{0} v_{0} e^{2 \Gamma t}$.

The second-order differential equation with real-valued coefficients (31) admits realvalued solutions $Q(t)$ upon appropriate initial conditions $Q(0), \dot{Q}(0) \in \mathbb{R}$. To write this in a more familiar form, we make $\mathcal{Q}=e^{-\Gamma t / 2} Q$ —a change of variable known as expanding coordinates $[63,64]$. Then,

$$
\ddot{\mathcal{Q}}+\left(w_{0}^{2}-\frac{\Gamma^{2}}{4}+4 \Omega_{0}^{2} e^{2 \Gamma t}\right) \mathcal{Q}=-2 \Omega_{0} v_{0} e^{3 \Gamma t / 2}
$$

is the equation of motion for a driven parametric oscillator with no damping.

- Constant mass: For $\Gamma=0$, the mass term in (29) is $m_{0}=$ const. In this case, the solutions of Equation (32) are immediate:

$$
\begin{gathered}
\mathcal{Q}(t)=\mathcal{A} \cos \left(\sqrt{w_{0}^{2}+4 \Omega_{0}^{2}} t+\varphi\right)-\frac{2 \Omega_{0} v_{0}}{w_{0}^{2}+4 \Omega_{0}^{2}}, \\
\dot{\mathcal{Q}}(t)=-\mathcal{A} \sqrt{w_{0}^{2}+4 \Omega_{0}^{2}} \sin \left(\sqrt{w_{0}^{2}+4 \Omega_{0}^{2}} t+\varphi\right),
\end{gathered}
$$

with $\mathcal{A}$ and $\varphi$ integration constants determined from the initial conditions as

$$
\mathcal{A}=\sqrt{\dot{\mathcal{Q}}^{2}(0)+\left(\mathcal{Q}(0)+\frac{2 \Omega_{0} v_{0}}{w_{0}^{2}+4 \Omega_{0}^{2}}\right)^{2}}, \quad \tan \varphi=-\frac{\sqrt{w_{0}+4 \Omega_{0}^{2}} \dot{\mathcal{Q}}(0)}{2 \Omega_{0} v_{0}+\left(w_{0}^{2}+4 \Omega_{0}^{2}\right) \mathcal{Q}(0)} .
$$

The dynamics on the configuration space $(\mathcal{Q}, \dot{\mathcal{Q}})$ describe elliptic closed trajectories, displaced along the $Q$ axis due to the presence of both $\Omega_{0}$ and $v_{0}$; see Figure 1 .

- $\quad$ Time-dependent mass. For $\Gamma \neq 0$, we may introduce the variable $z=e^{\Gamma t}$ to get

$$
z^{2} \frac{d^{2} \mathcal{Q}}{d z^{2}}+z \frac{d \mathcal{Q}}{d z}+\left(\Lambda^{2}+4{\overline{\Omega_{0}}}^{2} z^{2}\right) \mathcal{Q}=-2 \bar{v}_{0} \bar{\Omega}_{0} z^{3 / 2}
$$

where

$$
\Lambda^{2}:=\frac{w_{0}^{2}}{\Gamma^{2}}-\frac{1}{4}, \quad \bar{\Omega}_{0}=\frac{\Omega_{0}}{\Gamma}, \quad \bar{v}_{0}=\frac{v_{0}}{\Gamma} .
$$


We can immediately identify that the homogeneous part of (35) coincides with the Bessel differential equations [55]. In this case, we use the solutions

$$
\mathcal{Q}_{h ; 1}(t)=\frac{J_{i \Lambda}\left(2 \bar{\Omega}_{0} e^{\Gamma t}\right)}{\bar{\Omega}_{0}^{i \Lambda}}, \quad \mathcal{Q}_{h ; 2}(t) \equiv \mathcal{Q}_{h ; 1}^{*}(t)=\frac{J_{-i \Lambda}\left(2 \bar{\Omega}_{0} e^{\Gamma t}\right)}{\bar{\Omega}_{0}^{-i \Lambda}},
$$

where $J_{v}(z)$ is the Bessel function of the first kind, and the constants $\bar{\Omega}_{0}^{ \pm i \Lambda}$ have been introduced such that

$$
\lim _{\bar{\Omega}_{0} \rightarrow 0} \mathcal{Q}_{h ; 1}(t) \rightarrow \frac{\exp \left[i \sqrt{w_{0}^{2}-\Gamma^{2} / 4} t\right]}{\Gamma(1+i \Lambda)}, \quad \lim _{\bar{\Omega}_{0} \rightarrow 0} \mathcal{Q}_{h ; 2}(t) \rightarrow \frac{\exp \left[-i \sqrt{w_{0}^{2}-\Gamma^{2} / 4} t\right]}{\Gamma(1-i \Lambda)},
$$

lead to the solutions of the conventional oscillator.

On the other hand, after some calculations, the function

$$
\begin{aligned}
\mathcal{Q}_{p}(t)=i \frac{\pi \bar{v}_{0} \bar{\Omega}_{0}^{1-i \Lambda}}{2 \sinh (\pi \Lambda)} \frac{\Gamma\left(\frac{3}{4}-i \frac{\Lambda}{2}\right)}{\Gamma\left(\frac{7}{4}-i \frac{\Lambda}{2}\right) \Gamma(1-i \Lambda)} \times \\
\quad e^{\left(\frac{3}{2}-i \Lambda\right) \Gamma t} J_{i \Lambda}\left(2 \bar{\Omega}_{0} e^{\Gamma t}\right){ }_{1} F_{2}\left(\begin{array}{c}
\frac{3}{4}-i \frac{\Lambda}{2} \\
1-i \Lambda, \frac{7}{4}-i \frac{\Lambda}{2}
\end{array} \mid-\bar{\Omega}_{0}^{2} e^{2 \Gamma t}\right),
\end{aligned}
$$

provides the particular solution of Equation (35). Then, the general solution can be written in the form

$$
\mathcal{Q}(t)=\ell_{1} \operatorname{Re} Q_{h ; 1}(t)+\ell_{2} \operatorname{Im} \mathcal{Q}_{h ; 1}(t)+2 \operatorname{Re} \mathcal{Q}_{p}(t),
$$

where $\ell_{1}$ and $\ell_{2}$ are arbitrary real constants fixed from the initial conditions. In contrast to the constant-mass solutions, an explicit form for $\ell_{1}$ and $\ell_{2}$ in terms of the initial conditions $\mathcal{Q}(0)$ and $\dot{\mathcal{Q}}(0)$ is not feasible; however, it can be established by numerical means.

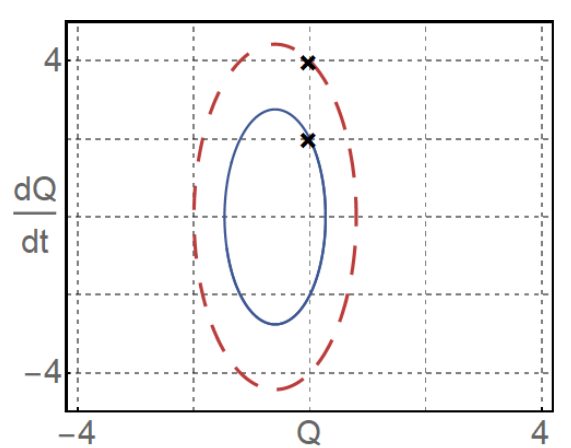

(a)

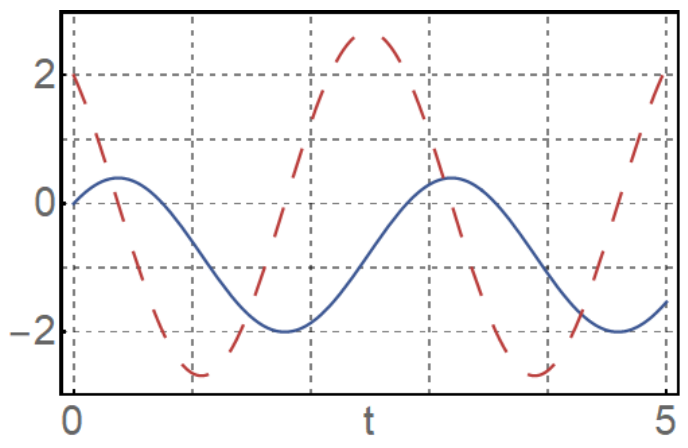

(b)

Figure 1. (a) Trajectories in the configuration space $(\mathcal{Q}, \dot{\mathcal{Q}})$ for constant-mass $(\Gamma=0)$, with $w_{0}=1$, $\Omega_{0}=1.5$, and $v_{0}=2$. The initial conditions are $\mathcal{Q}(0)=0, \dot{\mathcal{Q}}(0)=2$ (blue-solid), and $\mathcal{Q}(0)=0$, $\dot{\mathcal{Q}}(0)=4$ (red-dashed). The cross indicates the initial conditions. (b) The time-dependent functions $\mathcal{Q}$ (blue-solid) and $\mathcal{Q}$ (red-dashed) for the initial conditions $\mathcal{Q}(0)=0, \dot{\mathcal{Q}}(0)=2$.

The corresponding trajectory in the configuration space is depicted in Figure 2a for a finite time interval. Clearly, the trajectory is no longer closed as the particle mass is continuously changing over time. We remark that, as time passes, the particle localizes to a well-defined finite region that shrinks over time, while the velocity increases exponentially. This behavior is clear from Figure $2 b$. 


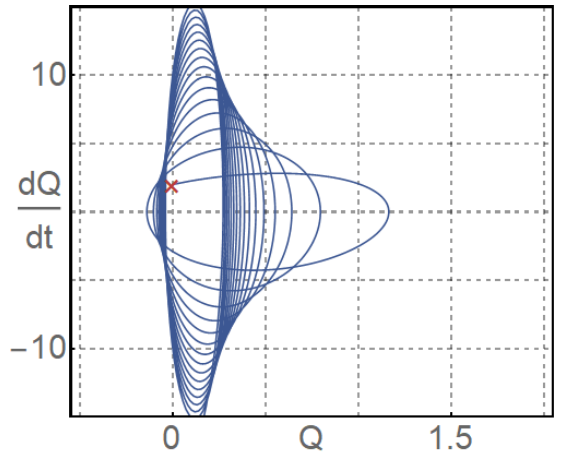

(a)

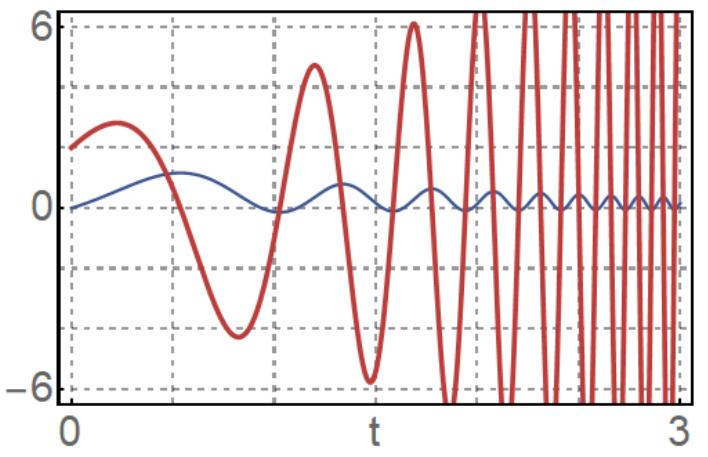

(b)

Figure 2. (a) Trajectories in the configuration space $(\mathcal{Q}, \dot{\mathcal{Q}})$ for $\Gamma=1$ in the interval $t \in(0,3)$, with $w_{0}=1, \Omega_{0}=1.5$, and $v_{0}=2$. The initial conditions are $\mathcal{Q}(0)=0$, and $\dot{\mathcal{Q}}(0)=2$. The red cross depicts the initial condition. (b) The time-dependent functions $\mathcal{Q}(t)$ (blue-solid) and $\dot{\mathcal{Q}}(t)$ (red-thick-solid) for the above-mentioned parameters.

\subsection{Quantum Picture}

The solutions of the Schrödinger equation defined by the Hamiltonian (28) require the functions $\sigma$ and $\gamma$. In this regard, the solutions of the homogeneous equation

$$
\ddot{q}+\left(w_{0}^{2}-\frac{\Gamma^{2}}{4}+4 \Omega_{0}^{2} e^{2 \Gamma t}\right) q=0
$$

serve to solve both the Ermakov and the inhomogeneous equations included in (23). Indeed, following [7,9], we know that the Ermakov equation defining $\sigma$ is solved by using the two linearly independent solutions of (41), namely $q_{1}$ and $q_{2}$. We thus obtain

$$
\sigma(t)=\left(a q_{1}^{2}(t)+b q_{1}(t) q_{2}(t)+c q_{2}^{2}(t)\right)^{\frac{1}{2}}, \quad b^{2}-4 a c=-\frac{w_{0}^{2}}{W_{0}^{2}},
$$

with $W_{0}=\operatorname{Wr}\left(q_{1}, q_{2}\right)$ the Wronskian of $q_{1}$ and $q_{2}$, which in this case is always a constant. In turn, the inhomogeneous equation associated with $\gamma$ shares solutions with the classical Equation (32), which was already solved in the previous section.

- For the constant mass case, we use the homogeneous solutions and their respective Wronskian $W_{0}$ as

$$
q_{1}=\cos \left(\sqrt{w_{0}^{2}+4 \Omega_{0}^{2}} t\right), \quad q_{2}=\sin \left(\sqrt{w_{0}^{2}+4 \Omega_{0}^{2}} t\right), \quad W_{0}=\sqrt{w_{0}^{2}+4 \Omega_{0}^{2}},
$$

whereas the solution for $\gamma(t)$ is the same as that for $-\mathcal{Q}(t)$ provided in (33).

- For the mass term $\mu(t)=e^{-\Gamma t / 2}$, we have

$$
q_{1}(t)=\operatorname{Re}\left(\frac{I_{i \Lambda}\left(2 \bar{\Omega}_{0} e^{\Gamma t}\right)}{\bar{\Omega}_{0}^{i \Lambda}}\right), \quad q_{2}(t)=\operatorname{Im}\left(\frac{I_{i \Lambda}\left(2 \bar{\Omega}_{0} e^{\Gamma t}\right)}{\bar{\Omega}_{0}^{i \Lambda}}\right), \quad W_{0}=\frac{\Gamma}{\pi} \sinh (\pi \Lambda),
$$

with $\bar{\Omega}_{0}$ and $\Lambda$ given in (36). Moreover, $\gamma(t)=-\mathcal{Q}(t)$, with $\mathcal{Q}(t)$ given in (40).

The profile of $\sigma(t)$ and $\gamma(t)$ is depicted in Figure 3, paying special attention to the constant mass $\Gamma=0$ and $\Gamma=1$ cases. In this figure, it is verified that the solution to the Ermakov equation is indeed always different from zero, as we stated earlier and proved in $[8,9]$ (see also [65]). Therefore, the point transformation is non-singular for $t \in \mathbb{R}$. 


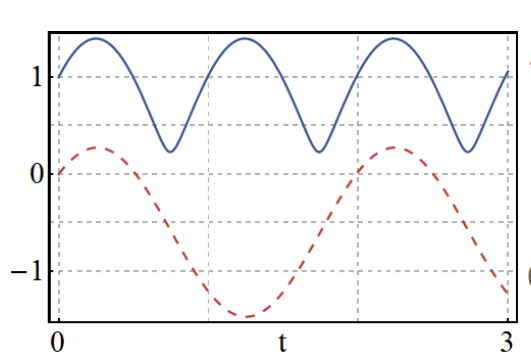

(a) $\Gamma=0$

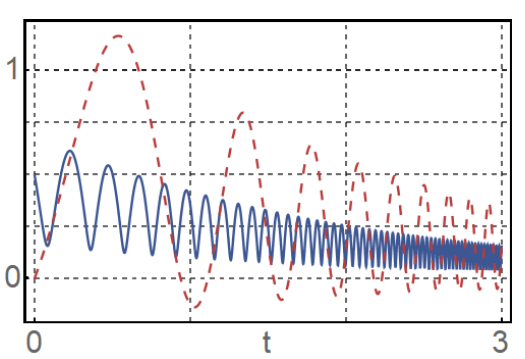

(b) $\Gamma=1$

Figure 3. Solution to the Ermakov equation $\sigma(t)$ (blue-solid) and the non-homogeneous equation $\gamma(t)$ (red-dashed) for the mass term $e^{-\Gamma t / 2}$ for the constant mass case $\Gamma=0$ (a) and $\Gamma=1(\mathbf{b})$. Moreover, in (a), we have used $\mathcal{A}$ and $\varphi$ as in Figure 1, whereas in (b), we have used $\ell_{1}$ and $\ell_{2}$ as in Figure 2. The remaining parameters have been selected in both cases as $a=c=w_{0}=1, \Omega=1.5, v_{0}=2$.

\subsection{Hermitian Conjugate and Bi-Orthogonality}

As a byproduct of the point transformation, the construction of the Hermitian conjugate $H_{\mathrm{sw}}^{\dagger}$ and its wave-functions is made obvious by noticing that

$$
H_{\mathrm{sW}}^{\dagger}=\frac{\hat{p}_{x}^{2}}{2 m_{0} \mu^{2}(t)}+m_{0} \mu^{2}(t) w^{2}(t) \hat{x}^{2}-\frac{i}{\mu^{2}(t)}\left(\Omega_{0}\left\{\hat{x}, \hat{p}_{x}\right\}+v_{0} \hat{p}_{x}\right) \neq H_{\mathrm{sW}}
$$

arises from $H_{\mathrm{sw}}$ through $\Omega_{0} \rightarrow-\Omega_{0}$ and $v_{0} \rightarrow-v_{0}$. This change leaves the differential equations defining $\sigma$ and $\gamma$ in (24) invariant. Therefore, the transformed coordinate $y(x, t)$ and time parameter $\tau(t)$ are the same for both $H_{\mathrm{sw}}$ and $H_{\mathrm{sw}}^{+}$. In this form, the solutions $\widetilde{\psi}(x, t)$ of the Schrödinger equation associated with (45) are also obtained from the stationary solutions

$$
\widetilde{\psi}(x, y)=(\widetilde{A}(x, t))^{-1} \Psi(y(x, t), \tau(t)), \quad \widetilde{A}(x, t)=\sqrt{\frac{\sigma}{\mu}} A_{0}(x, t)\left(\mathcal{A}_{1}(x, t)\right)^{-1} .
$$

That is,

$$
\widetilde{\psi}_{n}(x, t)=e^{-i E_{n} \tau(t) / \hbar} \widetilde{\phi}_{n}(x, t), \quad \widetilde{\phi}_{n}(x, t):=\sqrt{\frac{\mu}{\sigma}}\left(A_{0}(x, t)\right)^{-1} \mathcal{A}_{1}(x, t) \Phi_{n}(y(x, t)),
$$

with

$$
\Phi_{n}(y(x, t))=\sqrt{\frac{1}{2^{n} n !} \sqrt{\frac{m_{0} w_{0}}{\pi \hbar}}} e^{-\frac{m_{0} w_{0}}{2 \hbar}\left(\frac{\mu x+\gamma}{\sigma}\right)^{2}} H_{n}\left(\sqrt{\frac{m_{0} w_{0}}{\hbar}}\left(\frac{\mu x+\gamma}{\sigma}\right)\right) .
$$

The above results are remarkable since the wave-functions $\psi_{n}(x, t)$, together with their Hermitian conjugate counterparts $\widetilde{\psi}_{n}(x, t)$, form a bi-orthogonal system that provides a mathematical procedure to satisfy the superposition principle [8]. In this form, the nonHermitian oscillators introduced above can be studied in much the same way as in the Hermitian approaches. In the present case, the probability density may be studied through the following equivalent forms [10]:

$$
\mathcal{P}_{n}^{(B)}(x, t):=\left|\widetilde{\psi}_{n}^{*}(x, t) \psi_{n}(x, t)\right|=\left|\psi_{n}^{*}(x, t) \widetilde{\psi}_{n}(x, t)\right| .
$$

Figure 4 illustrates the time-evolution of the probability density (49) for the nonHermitian oscillators introduced in the previous section. In particular, for a constant mass, the probability density oscillates periodically with time; see the first row of Figure 4 . Note also that the wave packet width oscillates as well, producing a "breathing" effect as the wave packet propagates. This behavior matches well with the dynamics of the classical counterpart. For $\Gamma=1$, shown in the second row of Figure 4, the probability density is no longer periodic. Instead, the wave packet follows the trajectory of a damped oscillatory. 


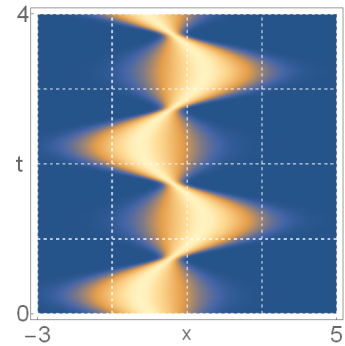

(a) $n=0$

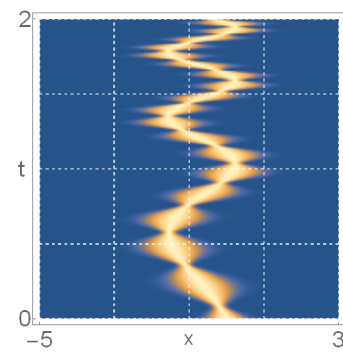

(d) $n=0$

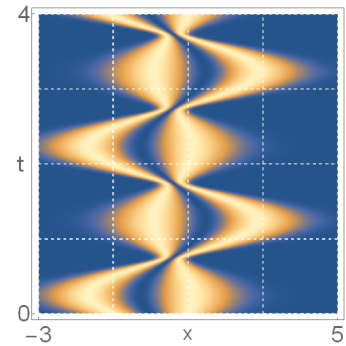

(b) $n=1$

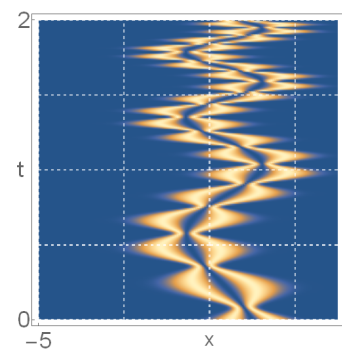

(e) $n=1$

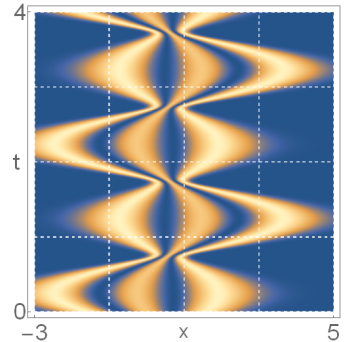

(c) $n=2$

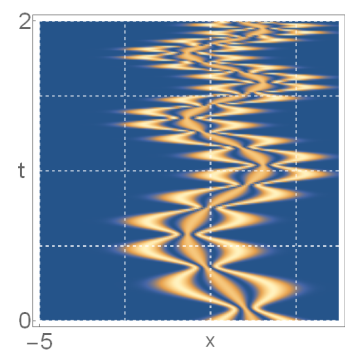

(f) $n=2$

Figure 4. Probability distribution in the bi-product sense (49) for the constant mass case $\Gamma=0$ (first row) and the time-dependent mass case $\Gamma=1$ (second row). The parameters are the same as in Figure 3.

\section{Conclusions}

In this manuscript, we have shown that the stationary harmonic oscillator is connected with time-dependent systems exhibiting non-Hermiticity via point transformations. Although this association includes a very wide set of such systems, we concentrated on a generalization of the Swanson oscillator that includes the Caldirola-Kanai system as particular case. We provided concrete expressions for the Hamiltonian and the corresponding exact solutions in both classical and quantum pictures. The systems studied here transit to their Hermitian configuration at the appropriate limit of the involved parameters. We have also shown that the point transformations automatically provide the Hermitian conjugate of the system under study as well as the corresponding solutions. This has been used to construct a bi-orthogonal system that allows the calculation of probability densities, which are dissimilar to the conventional densities in the sense that no phase shifts producing oscillations of the norm are allowed [10]. Noticeably, the real and imaginary parts of the fundamental solutions, as well as their probability densities, behave qualitatively equally in both normalizations, although the bi-normalized values are usually larger than the conventionally normalized values. The latter situation is reversed for superpositions of the basis elements (see [10]).

Author Contributions: Both authors contributed equally to this work. All authors have read and agreed to the published version of the manuscript.

Funding: This research has been funded by Consejo Nacional de Ciencia y Tecnología (CONACyT), Mexico, Grant No. A1-S-24569.

Institutional Review Board Statement: Not applicable.

Informed Consent Statement: Not applicable.

Data Availability Statement: Not applicable.

Acknowledgments: K. Zelaya acknowledges the support from the project "Physicist on the move II" (KINEÓ II), Czech Republic, Grant No. CZ.02.2.69/0.0/0.0/18_053/0017163. 
Conflicts of Interest: The authors declare no conflict of interest.

\section{Appendix A. Point Transformations}

In position-representation, the Schrödinger equation defined by the harmonic oscillator Hamiltonian (2) may be written as

$$
i \hbar \frac{\partial \Psi}{\partial \tau}=-\frac{\hbar^{2}}{2 m_{0}} \frac{\partial^{2} \Psi}{\partial y^{2}}+\frac{m_{0} w_{0}^{2}}{2} y^{2} \Psi .
$$

The solutions are provided in Equations (13) and (14) of the main text. Hereafter we say that the set $\{y, \tau, \Psi\}$ defines the frame of the harmonic oscillator $(\mathrm{HO})$, which is composited by the spatial-coordinates $y$, the time-variable $\tau$, and the solutions $\Psi$ of (A1).

Equivalently, for the operator $H_{\mathrm{sw}}(t)$ introduced in Equation (4) one has

$$
\begin{aligned}
i \hbar \frac{\partial \psi}{\partial t}=-\frac{\hbar^{2}}{2 m(t)} \frac{\partial^{2} \psi}{\partial x^{2}} & \\
& +(2 \hbar \Omega(t) x+\hbar v(t)) \frac{\partial \psi}{\partial x}+\left[\frac{m(t) w^{2}(t)}{2} x^{2}+F(t) x+\hbar \Omega(t)\right] \psi .
\end{aligned}
$$

The set $\{x, t, \psi\}$ defines the frame of the time-dependent non-Hermitian oscillator (TnH-HO), integrated by the spatial-coordinates $x$, the time-variable $t$, and the solutions $\psi$ of Equation (A2).

Within the point transformation theory [52], the mapping from $\mathrm{HO}$ to $\mathrm{TnH}-\mathrm{HO}$ is established by the relationships

$$
y=y(x, t), \quad \tau=\tau(x, t), \quad \Psi=\Psi(y(x, t), \tau(x, t))=G(x, t ; \psi(x, t)) .
$$

Computing the total derivatives $\frac{d \Psi}{d x}, \frac{d \Psi}{d t}$, and $\frac{d^{2} \Psi}{d x^{2}}$, one has

$$
\frac{\partial \Psi}{\partial \tau}=G_{1}\left(x, t ; \psi, \frac{\partial \psi}{\partial x}, \frac{\partial \psi}{\partial t}\right), \quad \frac{\partial^{2} \Psi}{\partial y^{2}}=G_{2}\left(x, t ; \psi, \frac{\partial \psi}{\partial x}, \frac{\partial^{2} \psi}{\partial x^{2}}, \frac{\partial \psi}{\partial t}\right) .
$$

To avoid nonlinear terms one may introduce the conitions [27]

$$
\Psi=G(x, t ; \psi)=A(x, t) \psi, \quad \tau=\tau(t) .
$$

After some calculations, from (A4) one arrives at

$$
\begin{aligned}
& \Psi_{\tau}=\frac{A}{\tau_{t}}\left[-\frac{y_{t}}{y_{x}} \psi_{x}+\psi_{t}+\left(\frac{A_{t}}{A}-\frac{y_{t}}{y_{x}} \frac{A_{x}}{A}\right) \psi\right], \\
& \Psi_{y, y}=\frac{A}{y_{x}^{2}}\left[\psi_{x, x}+\left(2 \frac{A_{x}}{A}-\frac{y_{x x}}{y_{x}}\right) \psi_{x}+\left(\frac{A_{x x}}{A}-\frac{y_{x x}}{y_{x}} \frac{A_{x}}{A}\right) \psi\right],
\end{aligned}
$$

the subindices denote partial derivatives, $f_{u}=\frac{\partial f}{\partial u}$. Substituting (A5) and (A6) into (A1) gives

$$
i \hbar \psi_{t}+\frac{\hbar^{2}}{2 m_{0}} \frac{\tau_{t}}{y_{x}^{2}} \psi_{x, x}+B(x, t) \psi_{x}-V(x, t) \psi=0,
$$

with

$$
\begin{aligned}
& B(x, t)=-i \hbar \frac{y_{t}}{y_{x}}+\frac{\hbar^{2}}{2 m_{0}} \frac{\tau_{t}}{y_{x}^{2}}\left(2 \frac{A_{x}}{A}-\frac{y_{x x}}{y_{x}}\right) \\
& V(x, t)=-i \hbar\left(\frac{A_{t}}{A}-\frac{y_{t}}{y_{x}} \frac{A_{x}}{A}\right)-\frac{\hbar^{2}}{2 m_{0}} \frac{\tau_{t}}{y_{x}^{2}}\left(\frac{A_{x x}}{A}-\frac{y_{x x}}{y_{x}} \frac{A_{x}}{A}\right)+\frac{\tau_{t}}{2} m_{0} w_{0}^{2} y^{2}(x, t) .
\end{aligned}
$$


Demanding coincidence of (A7) with (A2) requires

$$
\frac{\tau_{t}}{y_{x}^{2}}=\frac{m_{0}}{m(t)}, \quad B(x, t)=-2 \hbar \Omega(t) x .
$$

To simplify calculations we introduce real-valued functions $\mu(t)$ and $\sigma(t)$ such that $\tau_{t}=\sigma^{-2}(t)$ and $m(t)=m_{0} \mu^{2}(t)$. From the first condition in (A9) we get

$$
\tau(t)=\int^{t} \frac{d t^{\prime}}{\sigma^{2}\left(t^{\prime}\right)}, \quad y(x, t)=\frac{\mu(t) x+\gamma(t)}{\sigma(t)},
$$

where $\gamma(t): \mathbb{R} \rightarrow \mathbb{R}$ emerges as a constant of integration with respect to $x$. The above result is quoted as Equation (15) in the main text. On the other hand, the second condition in (A9) permits to determine the remaining transformation function $A(x, t)$, which we conveniently rewrite as

$$
A(x, t)=A_{0}(x, t) A_{1}(x, t),
$$

with

$$
A_{0}(x, t):=\exp \left[i \frac{m_{0}}{\hbar} \frac{\mu}{\sigma}\left(\frac{1}{2} \mathcal{W}_{\mu} x^{2}+\mathcal{W}_{\gamma} x+\xi_{1}\right)\right]
$$

and

$$
A_{1}(x, t):=\exp \left[-\frac{m_{0}}{\hbar} \mu^{2}\left(\Omega x^{2}+v x+\xi_{2}\right)\right] .
$$

The above expressions are quoted respectively as (19) and (20). The real-valued functions $\xi_{1}(t)$ and $\xi_{2}(t)$ are integration constants with respect to $x$, and

$$
\mathcal{W}_{\mu} \equiv \mathcal{W}_{\mu}(t)=\sigma \dot{\mu}-\dot{\sigma} \mu, \quad \mathcal{W}_{\gamma} \equiv \mathcal{W}_{\gamma}(t)=\sigma \dot{\gamma}-\dot{\sigma} \gamma,
$$

where $\dot{f}=\frac{d f}{d t}$. In this form, $A(x, t)$ is factorized as the product of the gauge transformation $A_{0}(x, t)$, working as a unitary transformation, and the non-gauge term $A_{1}(x, t)$, accounting for the non-Hermiticity of $H_{\mathrm{sw}}(t)$.

The explicit form of the time-dependent potential $V(x, t)$ introduced in (A8) is provided in Equation (16) of the main text, together with

$$
V_{0}(t)=m_{0}\left[\frac{d}{d t}\left(\frac{\mu}{\sigma} \xi_{1}\right)-\frac{\mathcal{W}_{\gamma}^{2}}{2 \sigma^{2}}-\frac{\mu^{2} v^{2}}{2}+\frac{w_{0}^{2} \gamma^{2}}{2 \sigma^{4}}+\frac{\hbar}{m_{0}} \Omega\right]+i m_{0}\left[\frac{d}{d t}\left(\mu^{2} \xi_{2}\right)-t \frac{\hbar}{2 m_{0}} \frac{\mathcal{W}_{\mu}}{\sigma \mu}\right]
$$

Comparing Equation (16) with the potential associated to the operator (4) gives a system of equations for $\sigma, \gamma, \xi_{1}$ and $\xi_{2}$. After making $V_{0}=\hbar \Omega^{-1}$ one gets

$$
\begin{gathered}
\ddot{\sigma}+\left[w^{2}+4 \Omega^{2}-\frac{\ddot{\mu}}{\mu}+\frac{2 i}{\mu^{2}} \frac{d}{d t}\left(\mu^{2} \Omega\right)\right] \sigma=\frac{w_{0}^{2}}{\sigma^{3}}, \\
\ddot{\gamma}+\left[w^{2}+4 \Omega^{2}-\frac{\ddot{\mu}}{\mu}+\frac{2 i}{\mu^{2}} \frac{d}{d t}\left(\mu^{2} \Omega\right)\right] \gamma=2 v \mu \Omega-\frac{i}{\mu} \frac{d}{d t}\left(\mu^{2} v\right),
\end{gathered}
$$

and

$$
\frac{d}{d t}\left(\frac{\mu}{\sigma} \xi_{1}+i \mu^{2} \xi_{2}-\frac{\gamma}{2 \sigma} \mathcal{W}_{\gamma}-i \frac{\hbar}{2 m_{0}} \ln \frac{\mu}{\sigma}\right)-i \frac{\gamma}{2 \mu} \frac{d}{d t} \mu^{2} v-\frac{\mu^{2} v^{2}}{2}+v \gamma \mu \Omega=0 .
$$

\section{References}

1. Bender, M.; Boettcher, S. Real Spectra in Non-Hermitian Hamiltonians Having PT Symmetry. Phys. Rev. Lett. 1998, 80, 5243. [CrossRef]

2. Lévai, G.; Znojil, M. Systematic search for PT-symmetric potentials with real energy spectra. J. Phys. A Math. Gen. 2000, $33,7165$. [CrossRef]

3. Geyer, H.; Heiss, D.; Znojil, M. (Eds.) Special Issue Dedicated to the Physics of Non-Hermitian Operators. In Proceedings of the PHHQP IV, Western Cape, South Africa, 23-25 November 2005; Volume 39. 
4. $\quad$ Fring, A.; Jones, H.; Znojil, M. (Eds.) Papers Dedicated to the Subject of the 6th International Workshop on Pseudo-Hermitian Hamiltonians In Quantum Physics (PHHQP VI) (City University, London, UK, 16-18 July 2007). J. Phys. A Math. Theor. 2008, 41, 240301-244027. [CrossRef]

5. Mostafazadeh, A. Pseudo-Hermiticity versus PT-Symmetry: The necessary condition for the reality of the spectrum of a nonHermitian Hamiltonian. J. Math. Phys. 2002, 43, 205. [CrossRef]

6. Mostafazadeh, A. Pseudo-Hermitian representation of Quantum Mechanics. Int. J. Geom. Methods Mod. Phys. 2010, 7, 1191. [CrossRef]

7. Rosas-Ortiz, O.; Castaños, O.; Schuch, D. New supersymmetry-generated complex potentials with real spectra. J. Phys. A Math. Theor. 2015, 48, 445302. [CrossRef]

8. Rosas-Ortiz, O.; Zelaya, K. Bi-Orthogonal Approach to Non-Hermitian Hamiltonians with the Oscillator Spectrum: Generalized Coherent States for Nonlinear Algebras. Ann. Phys. 2018, 388, 26. [CrossRef]

9. Blanco-García, Z.; Rosas-Ortiz, O.; Zelaya, K. Interplay between Riccati, Ermakov and Schrödinger equations to produce complex-valued potentials with real energy spectrum. Math. Meth. Appl. Sci. 2019, 42, 4925. [CrossRef]

10. Zelaya, K.; Dey, S.; Hussin, V.; Rosas-Ortiz, O. Nonclassical States for Non-Hermitian Hamiltonians with the Oscillator Spectrum. Quantum Rep. 2020, 2, 12. [CrossRef]

11. Zelaya, K.; Cruz y Cruz, S.; Rosas-Ortiz, O. On the construction of non-Hermitian Hamiltonians with all-real spectra through supersymmetric algorithms. In Geometric Methods in Physics XXXVIII; Trends in Mathematics; Birkhäuser: Cham, Switzerland, 2020; pp. 283-292.

12. Gbur, G.; Makris, K. Introduction to non-Hermitian photonics in complex media: PT-symmetry and beyond. Photonics Res. 2018, 6, PTS1. [CrossRef]

13. El-Ganainy, R.; Makris, K.G.; Christodoulides, D.N.; Musslimani, Z.H. Theory of coupled optical PT-symmetric structures. Opt. Lett. $200,32,2632$. [CrossRef]

14. Rüter, C.E.; Makris, K.G.; El-Ganainy, R.; Christodoulides, D.N.; Segev, M.; Kip, D. Observation of parity-time symmetry in optics. Nat. Phys. 2010, 6, 192. [CrossRef]

15. Mihalcea, B.M.; Filinov, V.; Syrovatka, R.; Vasilyak, L. The physics and applications of strongly coupled plasmas levitated in electrodynamic traps. arXiv 2019, arXiv:1910.14320.

16. Paul, W. Electromagnetic traps for charged and neutral particles. Rev. Mod. Phys. 1990, 62, 531. [CrossRef]

17. Combescure, M. A quantum particle in a quadrupole radio-frequency trap. Ann. Inst. Henri Poincare A 1986, $44,293$.

18. Pritchard, D.E. Cooling Neutral Atoms in a Magnetic Trap for Precision Spectroscopy. Phys. Rev. Lett. 1983, 51, 1336. [CrossRef]

19. Glauber, R.J. The Quantum Mechanics of Trapped Wavepackets. In Proceedings of the International Enrico Fermi Schoo, Course 118, Varenna, Italy, 1-19 July 1992; Arimondo, E., Philips, W.D., Sttrumia, F., Eds.; Societa Italia di Fisica: Morth Holland, The Netherlands, 1992; p. 643.

20. Mihalcea, B.M. A quantum parametric oscillator in a radiofrequency trap. Phys. Scr. 2009, 2009, 014006. [CrossRef]

21. Mihalcea, B.M.; Lynch, S. Investigations on Dynamical Stability in 3D Quadrupole Ion Traps. Appl. Sci. 2021, 11, 2938. [CrossRef]

22. Schwabl, F. Quantum Mechanics, 3rd ed.; Springer: Berlin/Heidelberg, Germany, 2002.

23. Lewis, H.R., Jr.; Riesenfeld, W.B. An Exact Quantum Theory of the Time-Dependent Harmonic Oscillator and of a Charged Particle in a Time-Dependent Electromagnetic Field. J. Math. Phys. 1969, 10, 1458. [CrossRef]

24. Bohm, A.; Mostafazadeh, A.; Koizumi, H.; Niu, Q.; Zwanziger, J. The Geometric Phase in Quantum Systems: Foundations, Mathematical Concepts, and Applications in Molecular and Condensed Matter Physics; Springer: Berlin/Heidelberg, Germany, 2003.

25. Dodonov, V.V.; Man'ko, O.V.; Man'ko, V.I. Quantum nonstationary oscillator: Models and applications. J. Russ. Laser Res. 1995, 16, 1. [CrossRef]

26. Guerrero, J.; López-Ruiz, F.F. On the Lewis-Riesenfeld (Dodonov-Man'ko) invariant method. Phys. Scr. 2015, 90, 074046. [CrossRef]

27. Zelaya, K.; Rosas-Ortiz, O. Quantum nonstationary oscillators: Invariants, dynamical algebras and coherent states via point transformations, Phys. Scr. 2020, 95, 064004. [CrossRef]

28. Dodonov, V.V. Invariant Quantum States of Quadratic Hamiltonians. Entropy 2021, 23, 634. [CrossRef]

29. Castaños, O.; Schuch, D.; Rosas-Ortiz, O. Generalized coherent states for time-dependent and nonlinear Hamiltonians via complex Riccati equations. J. Phys. A Math. Theor. 2013, 46, 075304. [CrossRef]

30. Cruz, H.; Schuch, D.; Castaños, O.; Rosas-Ortiz, O. Time-evolution of quantum systems via a complex nonlinear Riccati equation I. Conservative systems with time-independent Hamiltonian. Ann. Phys. 2015, 360, 44. [CrossRef]

31. Cruz, H.; Schuch, D.; Castaños, O.; Rosas-Ortiz, O. Time-evolution of quantum systems via a complex nonlinear Riccati equation II. Dissipative systems. Ann. Phys. 2016, 373, 690. [CrossRef]

32. Cruz y Cruz, S.; Gress, Z. Group approach to the paraxial propagation of Hermite-Gaussian modes in a parabolic medium. Ann. Phys. 2017, 383, 257. [CrossRef]

33. Ünal, N. Quasi-Coherent states for the Hermite oscillator. J. Math. Phys. 2018, 59, 062104. [CrossRef]

34. Zelaya, K.; Rosas-Ortiz, O. Comments on “Quasi-coherent states for the Hermite oscillator”. J. Math. Phys. 2019, 60, 054101. [CrossRef]

35. Cruz y Cruz, S.; Razo, R.; Rosas-Ortiz, O.; Zelaya, K. Coherent states for exactly solvable time-dependent oscillators generated by Darboux transformations. Phys. Scr. 2020, 95, 044009. [CrossRef] 
36. Zelaya, K.; Hussin, V. Time-dependent rational extensions of the parametric oscillator: Quantum invariants and the factorization method. J. Phys. A Math. Theor. 2020, 53, 165301. [CrossRef]

37. Zelaya, K.; Marquette, I.; Hussin, V. Fourth Painlevé and Ermakov equations: Quantum invariants and new exactly-solvable time-dependent Hamiltonians. J. Phys. A Math. Theor. 2021, 54, 015206. [CrossRef]

38. Bagrov, V.G.; Samsonov, B.F.; Shekoyan, L.A. Darboux transformation for the nonsteady Schrödinger equation. Russ. Phys. J. 1995, 38, 706. [CrossRef]

39. Zelaya, K.; Rosas-Ortiz, O. Exactly Solvable Time-Dependent Oscillator-Like Potentials Generated by Darboux Transformations. J. Phys. Conf. Ser. 2017, 839, 012018. [CrossRef]

40. Cen, J.; Fring, A.; Frith, T. Time-dependent Darboux (supersymmetric) transformations for non-Hermitian quantum systems. J. Phys. A Math. Theor. 2019, 52, 115302. [CrossRef]

41. Razo, R.; Cruz y Cruz, S. New confining optical media generated by Darboux transformations. J. Phys. Conf. Ser. $2019,1194,012091$. [CrossRef]

42. Contreras, A.; Jakubský, V. Photonic systems with two-dimensional landscapes of complex refractive index via time-dependent supersymmetry. Phys. Rev. A 2019, 99, 053812. [CrossRef]

43. Swanson, M.S. Transition elements for a non-Hermitian quadratic Hamiltonian. J. Math. Phys. 2004, 45, 585. [CrossRef]

44. Midya, B.; Dube, P.P.; Roychoudhury, R. Non-isospectrality of the generalized Swanson Hamiltonian and harmonic oscillator. J. Phys. A Math. Theor. 2011, 44, 062001. [CrossRef]

45. Graefe, E.M.; Korsch, H.J.; Rush, A.; Schubert, R. Classical and quantum dynamics in the (non-Hermitian) Swanson oscillator. J. Phys. A Math. Theor. 2015, 48, 055301. [CrossRef]

46. Bagchi, B.; Marquette, I. New 1-step extension of the Swanson oscillator and superintegrability of its two-dimensional generalization. Phys. Lett. A 2015, 379, 1584. [CrossRef]

47. Bagchi, B.; Banerjee, A.; Mandal, P. A generalized Swanson Hamiltonian in a second-derivative pseudo-supersymmetric framework. Int. J. Mod. Phys. A 2015, 30, 1550037. [CrossRef]

48. Dourado, R.A.; de Ponte, M.A.; Moussa, M.H.Y. A time-dependent pseudo-Hermitian Hamiltonian for a cavity mode with pure imaginary frequency. Phys. A Stat. Mech. Appl. 2021, 581, 126195. [CrossRef]

49. Fring, A.; Tenney, R. Exactly solvable time-dependent non-Hermitian quantum systems from point transformations. Phys. Lett. A 2021, 410, 127548. [CrossRef]

50. Lima, F.C.E.; Moreira, A.R.P.; Almeida, C.A.S. Information and thermodynamic properties of a non-Hermitian particle ensemble. Int. J. Quantum Chem. 2021, 121, e26645. [CrossRef]

51. Bagarello, F. Some results on the rotated infinitely deep potential and its coherent states. Phys. A Stat. Mech. Appl. 2021, 564, 125565. [CrossRef]

52. Steeb, W.H. Invertible Point Transformations and Nonlinear Differential Equations; World Scientific Publishing: Singapore, 1993.

53. Luiz, F.S.; de Ponte, M.A.; Moussa, M.H.Y. Unitarity of the time-evolution and observability of non-Hermitian Hamiltonians for time-dependent Dyson maps. Phys. Scrip. 2020, 95, 065211. [CrossRef]

54. Fring, A.; Moussa, M.H.Y. Non-Hermitian Swanson model with a time-dependent metric. Phys. Rev. A 2016, $94,042128$. [CrossRef]

55. Olver, F.W.J.; Lozier, D.W.; Boisvert, R.F.; Clark, C.W. (Eds.) NIST Handbook of Mathematical Functions; Cambridge University Press: New York, NY, USA, 2010.

56. Ermakov, V. Second order differential equations. Conditions of complete integrability. Kiev University Izvestia, Series III 9 (1880) 1. English translation by Harin A.O. Appl. Anal. Discret. Math. 2008, 2, 123. (In Russian) [CrossRef]

57. Schuch, D. Quantum Theory from a Nonlinear Perspective, Riccati Equations in Fundamental Physics; Springer: Cham, Switzerland, 2018.

58. Aldaya, V.; Cossío, F.; Guerrero, J.; López-Ruiz, F.F. The quantum Arnold transformation. J. Phys. A Math. Theor. 2011, 44, 065302. [CrossRef]

59. Zelaya, K.; Hussin, V. Point Transformations: Exact Solutions of the Quantum Time-Dependent Mass Nonstationary Oscillator. In Quantum Theory and Symmetries; Paranjape M.B., MacKenzie R., Thomova Z., Winternitz P., Witczak-Krempa W., Eds.; CRM Series in Mathematical Physics; Springer: Cham, Switzerland, 2021; pp. 295-303.

60. Caldirola, P. Forze non conservative nella meccanica quantistica. Nuovo Cim. 1941, 18, 393. [CrossRef]

61. Kanai, E. On the Quantization of the Dissipative Systems. Prog. Theor. Phys. 1948, 3, 440. [CrossRef]

62. Greenberger, D.M. A critique of the major approaches to damping in quantum theory. J. Math. Phys. 1979, 20, 762. [CrossRef]

63. Pedrosa, I.A. Canonical transformations and exact invariants for dissipative systems. J. Math. Phys. 1987, 28, 2662. [CrossRef]

64. Schuch, D. A new Lagrange-Hamilton formalism for dissipative systems. Int. J. Quantum Chem. 1990, 38, 767. [CrossRef]

65. Barbero, J.F.; Gómez Vergel, D.G.; Villaseñor, E.J.S. Evolution operators for linearly polarized two-Killing cosmological models. Phys. Rev. D 2006, 74, 024003. 